\section{Case Reports in Ophthalmology}

\title{
Solitary Idiopathic Choroiditis
}

\author{
Sérgio Monteiro Richard Andrews Mandeep Sagoo \\ Centro Hospitalar do Baixo Vouga, Aveiro, Portugal
}

\section{Key Words}

Solitary idiopathic choroiditis · Fundus autofluorescence · Optical coherence tomography

\begin{abstract}
Background: There are multiple conditions associated with the formation of a solitary choroidal granuloma. However, in many cases, solitary choroiditis remains idiopathic in spite of an extensive systemic evaluation. Methods: A 26-year-old man presented with an asymptomatic pale choroidal lesion that had the features of solitary idiopathic choroiditis. Results: Optical coherence tomography and fundus autofluorescence were performed and showed lesion features. Conclusions: Solitary idiopathic choroiditis is a rare condition of unknown aetiology that sometimes can be mistaken as an intraocular tumour. The use of emerging multimodal imaging is of great importance in the diagnosis of this condition.
\end{abstract}

(C) 2014 S. Karger AG, Basel

\section{Introduction}

Solitary idiopathic choroiditis (SIC) is a rare condition described for the first time in 1997. The diagnosis is based on the funduscopic appearance of a solitary choroidal granuloma and the findings on multimodal imaging such as fundus autofluorescence (FAF) and optical coherence tomography (OCT). The use of these methods is of great importance in the differentiation of SIC from more serious conditions (systemic diseases and tumours).

\section{Case Report}

An asymptomatic 26-year-old previously healthy white male was referred to us following a routine optometrist visit. Visual acuity was $6 / 6$ in both eyes, with no sign of anterior 
Monteiro et al.: Solitary Idiopathic Choroiditis

segment inflammation, but with the presence of a single, inactive, yellow choroidal lesion, $0.75 \mathrm{~mm}$ in diameter, located inferior to the left optic disc (fig. 1).

Time domain OCT of the lesion showed thinning and compression of the overlying choroidal vasculature with an apparently normal retina above it. Spectral domain OCT with enhanced depth imaging (fig. 2) helped locate the lesion on the choroid and sclera, under the attenuated choroidal vasculature, without evidence of disruption of the outer or inner retinal layers and the retinal pigment epithelium (RPE). FAF (fig. 3) revealed a hyperautofluorescent area corresponding with the mass.

Ultrasound B-scans failed to show any elevated lesion or thickening of the 'coats' since conventional B-scans cannot separate choroidal from scleral layers. Also, there was no evidence of the sub-Tenon fluid and the T-sign was negative.

The common systemic causes of a choroidal granuloma were tested for sarcoidosis (serum angiotensin-converting enzyme and calcium) and tuberculosis (QuantiFERON Gold), and both were negative. Other less common causes of SIC such as Toxocara, Brucella and Wegener's disease were excluded either by anamnesis and/or additional tests. Also, inflammatory markers (erythrocyte sedimentation rate and C-reactive protein) were within normal limits and the chest X-ray showed no abnormalities. Taking the funduscopic appearance and the image characteristics on the OCT and FAF into account, the diagnosis of SIC was made.

\section{Discussion}

There are multiple conditions associated with the formation of a solitary choroidal granuloma [1]. With or without signs of generalized ocular inflammation, those lesions can be seen in the context of sarcoidosis, tuberculosis, toxocariasis and many other diseases [2]. However, in many cases, solitary choroiditis remains idiopathic in spite of an extensive systemic evaluation [1]. Described for the first time in 1997 by Hong et al. [3] in a series of 6 patients with a peculiar choroidal lesion that was at the time called unifocal helioid choroiditis, and characterized by a solitary, yellow-white choroidal mass of one disc diameter in basal dimension, this condition was later renamed SIC by Shields et al. [1] when they presented a series of 60 patients in 2002 (the largest to date).

The diagnosis of SIC, as described by Shields et al. [1], is based on the typical ophthalmoscopic features that vary depending on whether the lesion is active or inactive. In the inactive stage, SIC appears as a discrete, nummular, yellow-white lesion. An ill-defined, redorange halo surrounding the lesion is a highly characteristic sign. In this stage, retinochoroidal shunt vessels can occasionally be present and retinal pigment epithelial changes are subtle. When active, SIC lesions appear as a dull-yellow choroidal lesion with an ill-defined margin, yellow intraretinal exudation, localized subretinal fluid and occasional retinal vascular dilation as well as focal retinal haemorrhages. As inflammation resolves, the margins usually become better defined and exudation, haemorrhage, subretinal fluid and vascular abnormalities disappear.

SIC can occur at any age, but most patients are diagnosed between the third and sixth decades of their life. In the Shields et al. [1] series, it was more common in white (93\%) female $(63 \%)$ patients with no predilection for laterality. At the time of presentation, most patients are symptomatic: all 6 patients in the Hong et al. [3] series and 39 patients (65\%) in the Shields et al. [1] series. The most common presenting symptoms are blurred vision (38\%), floaters (15\%), mild pain (5\%), metamorphopsia (3\%) and scotoma (3\%). The most common location for SIC lesions is posterior to the equator $[1,3]$. The visual acuity is usually 
good, unless the lesion affects the juxtapapillary or foveal areas. The intraocular pressures are normal and there are generally no signs of anterior segment inflammation. In the case described here, the patient is a Caucasian male with no visual symptoms and the lesion located posterior to the equator.

The appearance of the lesion on the fluorescein angiogram also varies with the grade of inflammation. An active lesion shows hypofluorescence in the vascular filling phases and progressive hyperfluorescence in the later phases, with an ill-defined margin due to the leakage into the subretinal fluid and vitreous. An inactive lesion also shows early relative hypofluorescence and intense late staining, but with a well-defined margin.

Recently, Fung et al. [4] presented a series of 10 patients with SIC where the FAF, infrared reflectance (IR) imaging and the enhanced depth imaging OCT (EDI OCT) were reviewed. They concluded that the FAF findings are variable, with homogenous hyperautofluorescence being the most common finding, which is in agreement with our case. Fundus hyperautofluorescence is usually the result of accumulation of lipofuscin in diseased RPE [5]. However, Fung et al. [4] found only 1 case (out of 10) with abnormal RPE thickness on EDI OCT. They postulated that the hyperautofluorescence of these lesions is the result of unmasking, underlying scleral autofluorescence since bare sclera is known to demonstrate mild hyperautofluorescence. Another cause can be the thinned choroid above all the lesions in that series.

The most common finding on IR was a nearly uniformly hyperreflective area (9 out of 10 patients) [4]. Imaging with IR reflects the presence of melanin, collagen or fibrin [6]. Melanin is unlikely in these patients given the hypopigmented colour of SIC lesions, and fibrin is unlikely because it is usually found in the subretinal space and was not seen in any patient on EDI OCT. Fung et al. [4] postulated the IR hyperreflective findings may relate to an unmasking of scleral collagen without any interference from the overlying choroidal pigment.

The EDI OCT findings have enabled a more accurate localization of these lesions. Previous reports have suggested that SIC is confined to the choroid [1,3]. On the contrary, EDI OCT in combination with the findings on FAF and IR imaging suggest that it may also extend into the sclera. In all 10 cases of the Fung et al. [4] series, the SIC lesion was less reflective than the normal sclera and appeared to extend to the deepest parts of the EDI OCT scans. In all cases, there was thinning or compression of the overlying choroidal vasculature, and the posterior margin was only visible in 1 lesion. EDI OCT also showed the disruption of the outer retina and the RPE in some cases, but without any inner retinal disruption. It was assumed that the disruption of the outer retina and the RPE with sparing of the inner retina probably related to a choroidal thinning, with subsequent diminished oxygen and nutrient supply [4]. Ultrasonography is of little diagnostic value since the lesions are generally very small and shallow [1]. The aetiology of SIC is not known and the differential diagnosis includes all the conditions that can cause a choroidal granuloma: sarcoidosis [7-10], tuberculosis [11-17], ocular toxocariasis [18], and cat-scratch disease [19] among others. In fact, since gamma-interferon testing was not available at the time Shields et al. [1] published their series, we can speculate that some of the SIC cases could be manifestations of extrapulmonary tuberculosis and so anti-tuberculous therapy would be justified in cases with active choroidal lesions with markedly raised QuantiFERON titres.

Most of these conditions can be diagnosed by clinical findings and laboratory test results. None of the reported patients had any findings suggestive of any other ocular disease $[1,3]$. Other ocular inflammatory conditions that can remotely resemble SIC include ocular histoplasmosis, ocular toxoplasmosis, nodular posterior scleritis and syphilis, but again, there are features that permit a differentiation from SIC. 
Monteiro et al.: Solitary Idiopathic Choroiditis

Ocular tumors such as amelanotic choroidal melanoma, amelanotic choroidal naevus, choroidal metastasis, solitary choroidal hemangioma, choroidal osteoma, lymphoma, retinoblastoma and retinal astrocytic hamartoma may be clinically similar to SIC [1, 20-24]. The use of emerging multimodal imaging is of great importance in the differentiation of these conditions from SIC.

The management of SIC depends on the inflammation level. Active lesions demand a systemic evaluation for the aforementioned specific lesions and, when located in the region of the optic disc or fovea, should be treated with systemic corticosteroids. When the lesion appears inactive in an asymptomatic patient, evaluation should be limited to testing for treatable conditions like sarcoidosis, syphilis and tuberculosis. If positive results are found, a course of appropriate treatment must be considered, otherwise periodic observation is sufficient.

\section{References}

1 Shields JA, Shields CL, Demirci H, Hanovar S: Solitary idiopathic choroiditis: the Richard B. Weaver lecture. Arch Ophthalmol 2002;120:311-319.

2 Nussenblatt RB, Whitcup SM, Palestine AG: Uveitis; in Nussenblatt RB, Whitcup SM, Palestine AG (eds): Fundamentals and Clinical Practice. St. Louis, Mosby-Year Book Inc, 1996, pp 155-385.

-3 Hong PH, Jampol LM, Dodwell DG, et al: Unifocal helioid choroiditis. Arch Ophthalmol 1997;115:1007-1013.

-4 Fung AT, Kaliki S, Shields CL, Mashayekhi A, Shields JA: Solitary idiopathic choroiditis: findings on enhanced depth imaging optical coherence tomography in 10 cases. Ophthalmology 2013;120:852-858.

-5 Spaide RF: Fundus autofluorescence and age-related macular degeneration. Ophthalmology 2003;110:392399.

6 Theelen T, Hoyng CB, Klevering BJ: Near-infrared subretinal imaging in choroidal neovascularization; in Holz FG, Spaide RF (eds): Medical Retina: Focus on Retinal Imaging [Krieglstein GK, Weinreb RN, series eds. of Essentials in Ophthalmology]. New York, Springer, 2010, pp 79-80.

7 Marcus DF, Bovino JA, Burton TC: Sarcoid granuloma of the choroid. Ophthalmology 1982;89:1326-1330.

8 Olk RJ, Lipman MJ, Cundiff HC, Daniels J: Solitary choroidal mass as the presenting symptom in systemic sarcoidosis. Br J Ophthalmol 1983;67:826-829.

-9 Campo RV, Aaberg TM: Choroidal granuloma in sarcoidosis. Am J Ophthalmol 1984;97:419-427.

10 Tingey DP, Gonder JR: Ocular sarcoidosis presenting as a solitary choroidal mass (review). Can J Ophthalmol 1992;27:25-29.

11 Shields JA, Shields CL: Differential diagnosis of posterior uveal melanoma: chorioretinal granuloma; in: Intraocular Tumors: A Text and Atlas. Philadelphia, WB Saunders Company, 1992, pp 145-146.

12 Shields JA, Shields CL: Lesions simulating posterior uveal melanoma: choroidal granuloma; in: Atlas of Intraocular Tumors. Philadelphia, Lippincott Williams \& Wilkins, 1999, pp 146-147.

13 Cangemi FE, Friedman AH, Josephberg R: Tuberculoma of the choroid. Ophthalmology 1980;97:252-256.

14 Smith RE: Tuberculoma of the choroid (discussion). Ophthalmology 1980;87:256-257.

15 Jabbour NM, Faris B, Trempe CL: A case of pulmonary tuberculosis presenting with choroidal tuberculoma. Ophthalmology 1985;92:834-837.

16 Lyon CE, Grimson BS, Peiffer RL Jr, Merritt JC: Clinicopathological correlation of a solitary choroidal tuberculoma. Ophthalmology 1985;92:834-837.

17 Mansour AM, Haymond R: Choroidal tuberculomas without evidence of extraocular tuberculosis. Graefhes Arch Clin Exp Ophthalmol 1990;228:382-385.

18 Shields JA: Ocular toxocariasis: a review. Surv Ophthalmol 1984;28:361-381.

19 Pollock SC, Kristinsson J: Cat-scratch disease manifesting as unifocal helioid choroiditis. Arch Ophthalmol 1998:116:1249-1251.

20 Shields JA, Shields CL: Clinical features of posterior uveal melanoma: choroidal granuloma; in: Atlas of Intraocular Tumors. Philadelphia, Lippincott Williams \& Wilkins, 1999, pp 74-93.

-21 Brown GC, Shields JA, Augsburger JJ: Amelanotic choroidal nevi. Ophthalmology 1981;88:1116-1121.

-22 Shields CL, Shields JA, Gross NE, Schwartz GP, Lally SE: Survey of 520 uveal metastases. Ophthalmology 1997;104:1265-1276.

23 Shields CL, Shields JA, Augsburger JJ: Choroidal Osteoma (review). Surv Ophthalmol 1988;33:17-27.

24 Shields JA, Shields CL: Intraocular lymphoid tumors and leukemias; in: Atlas of Intraocular Tumors. Philadelphia, Lippincott Williams \& Wilkins, 1999, pp 324-330. 
Case Reports in

Ophthalmology

\begin{tabular}{l|l}
\hline Case Rep Ophthalmol 2014;5:1-5 \\
\hline DOI: 10.1159/000357470 & $\begin{array}{l}\text { ○ 2014 S. Karger AG, Basel } \\
\text { www.karger.com/cop }\end{array}$ \\
\hline
\end{tabular}

Monteiro et al.: Solitary Idiopathic Choroiditis

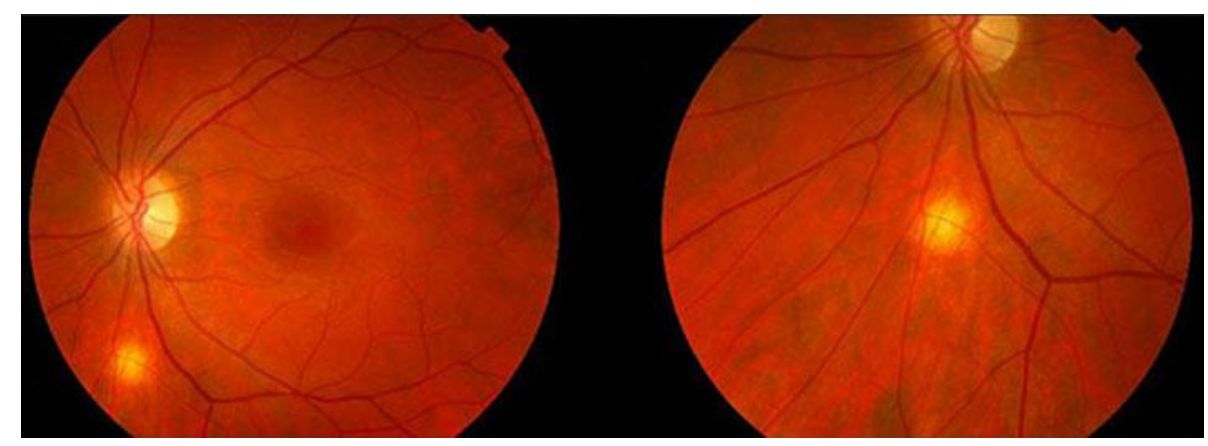

Fig. 1. Funduscopic appearance of the lesion. Note the yellow mass inferior to the optic disc.

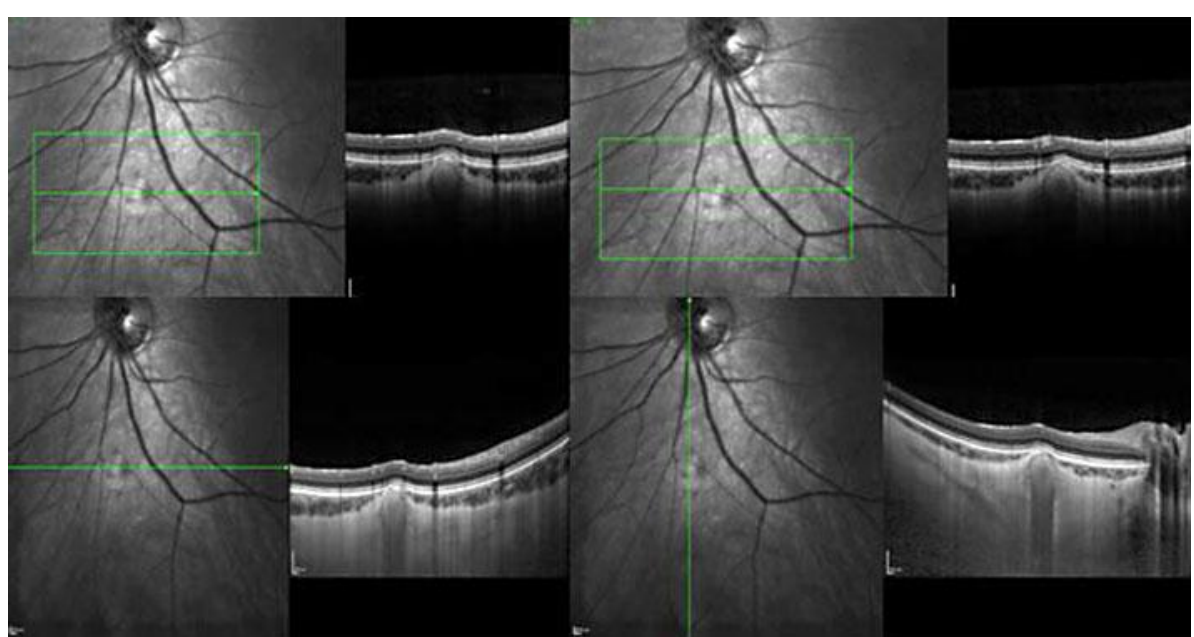

Fig. 2. Left eye: time domain OCT scan showing the thinning in the choroidal vessels above the lesion.

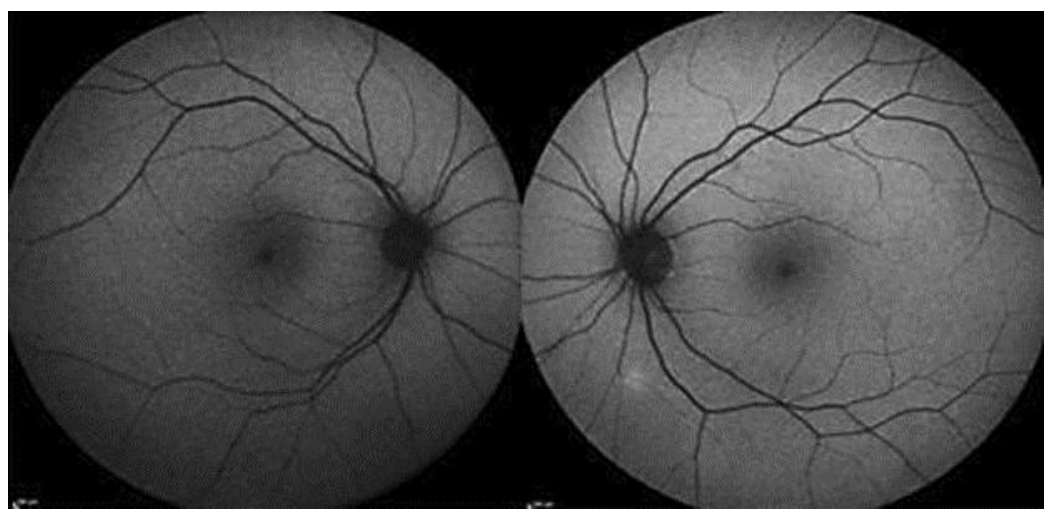

Fig. 3. Spectral domain OCT outlining the location of the lesion and the choroidal thinning with no evidence of disruption of the outer or inner retinal layers and RPE. 